\title{
Um levantamento bibliométrico do uso de imagens na prática da comunicação da governança pública em portais governamentais municipais
}

A comunicação do governo com seus cidadãos e o avanço das ferramentas de informação e contribui para que o Estado busque continuamente uma maior interação e proximidade com a população através de seus portais governamentais na internet. Dessa forma, a comunicação pública está diretamente associada à governança pública, representada pelo Estado executor de políticas públicas. E a manifestação de imagens nas plataformas governamentais (sites) é objeto de estudo da semiótica, uma vez que está é quem organiza a produção e compressão do sentido da linguagem não verbal. O objetivo deste trabalho é realizar um levantamento bibliométrico de publicações nacionais e internacionais concernentes ao uso de imagens na prática da comunicação da governança pública nos portais governamentais dos municípios brasileiros e de outros países. A área de conhecimento do estudo é a Administração Pública (Business, Management and Accounting). A abordagem metodológica se utiliza da teoria bibliométrica e os procedimentos de coleta de dados ocorrem junto às bases Eric - Educational Resources Information Centre, Google Scholar, Science Direct, Scopus e Web of Science. Quanto aos seus objetivos, a pesquisa é definida como exploratóriodescritiva, e definida como aplicada, quanto à sua natureza. As análises conduzidas demonstram que, com o emprego da totalidade dos termos de pesquisa, quais são Public Communication, Public Governance, Semiotics e Image(s), nenhum artigo foi encontrado nas cinco bases utilizadas. Embora mereça destaque o volume significativo de artigos que retornaram a partir da busca com emprego de frações dos termos de pesquisa, trata-se de produções científicas alheias ao objeto de pesquisa. Os resultados sugerem que esse déficit científico apontado no presente trabalho pode ser revelador de uma menor percepção dos cidadãos em relação ao conteúdo dos portais governamentais dos municípios. Ademais, face a amplitude de significação do campo de estudo da semiótica, os resultados também sugerem que não seria pelo aumento do número de bases de dados ou robustez da amostra, que a produção científica seja mais evidente em levantamentos bibliográficos dessa natureza.

\section{A bibliometric survey of the use of images in the practice of public governance communication in municipal government portals}

\begin{abstract}
The government's communication with its citizens and the advancement of information tools and contributes to the State's continuous search for greater interaction and proximity with the population through its governmental internet portals. Thus, public communication is directly associated with public governance, represented by the State that executes public policies. And the manifestation of images on government platforms (websites) is the object of study of semiotics, since it is who organizes the production and compression of the sense of non-verbal language. The objective of this work is to carry out a bibliometric survey of national and international publications concerning the use of images in the practice of public governance communication in the government portals of Brazilian municipalities and other countries. The study's area of knowledge is Public Administration (Business, Management and Accounting). The methodological approach uses bibliometric theory and the data collection procedures occur along the Eric - Educational Resources Information Center, Google Scholar, Science Direct, Scopus and Web of Science databases. As for its objectives, the research is defined as exploratory-descriptive, and defined as applied, as to its nature. The analyzes conducted show that, with the use of all the search terms, which are Public Communication, Public Governance, Semiotics and Image (s), no article was found in the five databases used. Although it is worth mentioning the significant volume of articles that returned from the search using fractions of the search terms, these are scientific productions alien to the object of research. The results suggest that this scientific deficit pointed out in the present study may reveal a lesser perception of citizens in relation to the content of the governmental portals of the municipalities. Furthermore, given the breadth of significance of the semiotics field of study, the results also suggest that it would not be due to the increased number of databases or the robustness of the sample, that scientific production is more evident in bibliographic surveys of this nature.
\end{abstract}

Keywords: Public governance; Public communication; Images; Semiotics; Bibliometric survey.

Topic: Gestão Pública

Reviewed anonymously in the process of blind peer.

Marcio do Nascimento Martins

Universidade Tecnológica Federal do Paraná, Brasil

http://lattes.cnpq.br/5466212558658375

http://orcid.org/0000-0002-7499-5667

marcionmartins@gmail.com

Ivan Carlos Vicentin (iD)

Universidade Tecnológica Federal do Paraná, Brasil

http://lattes.cnpq.br/6534550312384359

http://orcid.org/0000-0001-9039-0046

vicentini@utfpr.edu.br
Received: 07/04/2021

Approved: 04/06/2021
6

DOI: 10.6008/CBPC2179-684X.2021.002.0009
Referencing this:

MARTINS, M. N.; VICENTIN, I. C.. Um levantamento bibliométrico do uso de imagens na prática da comunicação da governança pública em portais governamentais municipais. Revista Brasileira de Administração Científica, v.12, n.2, p.100-112, 2021. DOI: http://doi.org/10.6008/CBPC2179-684X.2021.002.0009 


\section{INTRODUÇÃO}

Com o avanço das ferramentas de tecnologia de informação e comunicação (TICs), o Estado passou também a se utilizar dessas tecnologias na comunicação pública, buscando a interação e proximidade com a população. A comunicação pública, por sua vez, é um processo que se instaura na esfera pública entre o Estado, o governo e a sociedade e que se propõe a ser um espaço privilegiado de negociação entre os interesses das diversas instâncias de poder constitutivas da vida pública social (DUARTE, 2012).

Há uma perspectiva de que o uso de imagens está se tornando cada vez mais importante e mais utilizada. Por outro lado, há um movimento em prol da governança, que mostra a relevância da gestão pública e da comunicação pública. Existe também uma demanda crescente de governança pública no setor público e uma necessidade de se comunicá-la que, na qual, a própria transparência exige. E, por fim, há uma tendência cada vez maior do uso de imagens na comunicação.

Não são as novas tecnologias, mas as mudanças nos contextos sociais, que determinam as transformações nos movimentos sociais. As demandas dos agentes políticos encontram canais de manifestação e organização nas redes sociais, embora tais avanços tecnológicos tragam desafios (TILLY et al., 2010). Mesmo simples acessos ou visualizações de conteúdo desencadeiam os mecanismos de seleção, e os usuários estão cada vez mais conscientes de que fazem parte de uma rede que reproduz seu modo de existir (PEDRONI et al., 2014).

A necessidade de comunicação do governo com seus cidadãos, assim como a ocorrência da linguagem não verbal nas plataformas dos portais municipais, tem mostrado que é tarefa da semiótica organizar essas categorias e essas operações abstratas, que estão na base de nossa produção e compressão do sentido (TATIT, 2001).

O apanhado de comunicação pública para o contexto desta pesquisa recai na comunicação pública utilizada pelos governos municipais em seus portais governamentais na internet. E as organizações públicas precisam também utilizar-se desta tecnologia, principalmente das mídias sociais, para informar ao seu público de interesse as ações que desenvolvem e, assim, formar e transformar a opinião pública (DECKERT, 2017). Nesse contexto, emerge-se, portanto, a questão sobre qual é o padrão para se comunicar governança pública, utilizando-se dos recursos da semiótica, na prática da comunicação da governança pública nos portais governamentais das prefeituras na internet.

Uma vez que a técnica bibliométrica é conceituada na quantificação dos processos de comunicação escrita, esta pesquisa tem por objetivo realizar um levantamento bibliográfico das publicações existentes na literatura que tratam da produção científica acerca do uso de imagens na prática da comunicação da governança pública nos portais governamentais (sites das prefeituras), tanto dos municípios brasileiros, quanto de outros países.

Justifica-se, portanto, que o método bibliométrico utilizado tem por objetivo localizar artigos com essa identidade de conteúdo e propósito, assim como propiciar sua replicação e reprodução na ciência. Tal processo busca identificar os principais temas tratados na área, tendências e lacunas, bem como os principais 
autores e obras (WATANUKI et al., 2014).

Além desta seção introdutória, o artigo está estruturado pelo referencial teórico, abordando os tópicos imagens, semiótica, comunicação pública, governança pública e pesquisa bibliométrica. Posteriormente são descritos os procedimentos metodológicos adotados, a caracterização da pesquisa e o procedimento e coleta e análise dos dados. Em seguida, são apresentados a análise discussão dos resultados. Por fim, apresenta-se a conclusão acerca dos resultados obtidos.

\section{REVISÃO TEÓRICA}

Imagens, semiótica, comunicação pública e governança pública nos portais governamentais da internet

O conceito de governança inicialmente estava restrito à noção de desempenho gerencial e administrativo. Esse termo governança surge tendo como referência a concepção de bom governo (good government) e na competência do Estado de executar de forma consistente as políticas públicas (MATIASPEREIRA, 2010).

Uma boa governança pública, à semelhança da corporativa, está apoiada em quatro princípios: relações éticas; conformidade, em todas as suas dimensões; transparência; e prestação responsável de contas. A ausência desses princípios requer mudança na forma de gestão. A busca permanente da prática dos princípios na administração do país ou na gestão de qualquer instituição deve ser vista como uma condição indispensável para que essa instituição possa continuar a progredir (MATIAS-PEREIRA, 2014).

E, para colocar em prática esses princípios, há que se entender a fundo o verdadeiro sentido da comunicação pública, que é o do interesse público, cuja expressão é, frequentemente, empregada para substituir bem comum. Dentro da dimensão da comunicação da instituição pública, inclui-se a comunicação para promoção da imagem. Haswani (2013) observa que essa modalidade de comunicação utiliza todas as técnicas colocadas em prática pela publicidade comercial para envolver o público. Ela objetiva "conquistar confiança e aprovação dos cidadãos seja para angariar colaboração com ações do governo, seja para obtenção de futuro sucesso eleitoral" (HASWANI, 2013).

O bem comum seria a reunião de todas aquelas condições, no âmbito da vida social, que favoreçam o desenvolvimento da personalidade humana e com ela consistam. Daí não se adotar uma única corrente como suporte teórico para tratar da comunicação governamental, mas extrair de cada uma as peças necessárias à construção dos argumentos (DALLARI, 2003).

Os processos de comunicação não são apenas interativos, mas os são porque implicam as mediações simbólicas presentes nos complexos programas computacionais. A semiótica é a ciência geral que se encarrega de todas as linguagens e técnica da leitura de todos os signos. É a ciência que tem por objeto de investigação todas as linguagens possíveis, ou seja, que tem por objeto o exame dos modos de constituição de todo e qualquer fenômeno de produção de significação e de sentido (SANTAELLA, 1992).

De acordo com Souza et al. (2008), a imagem de anúncios publicitários como objeto de análise vem sendo realizada desde os anos 1960, principalmente por meio de uma teoria conhecida como semiótica. 
Roland Barthes foi o primeiro teórico da semiótica a se interessar pela análise publicitária e das comunicações e paralelamente com Umberto Eco contribuíram para os estudos de significação da imagem nessa primeira etapa.

Em uma segunda fase, autores como Jacques Durand e Georges Péninou, na década de 1970, deram conta de complementar os trabalhos de Barthes em relação à criação de uma retórica da imagem publicitária (SOUZA et al., 2008).

Nos anos 1980 e 1990, a busca pelos detalhes do tipo de discurso e dos mecanismos de persuasão, Floch, analisa logotipos, campanhas e anúncios constituindo a terceira fase. No final desse período, temos a atuação de Martine Joly fazendo releituras das outras fases utilizando a semiótica de Peirce e cria categorias para analisar a imagem. Andréa Semprini, que juntamente com Joly são considerados ícones da quarta fase, se utilizando das ideias de Floch e analisa imagens campanhas de forma diacrônica (SOUZA et al., 2008).

A semiótica, embora seja relativamente consensual que se trata de uma ciência dos signos, as definições de signos diferem de uma escola para a outra. Além da heterogeneidade que lhe é característica, também há uma diversidade de níveis em que a semiótica é concebida como ciência (SANTAELLA, 2004).

A palavra imagem, frequentemente utilizada nos estudos de semiótica plástica, é polissémica, por isso gera ambiguidades indesejáveis no discurso científico. Fala-se em imagem da fotografia, da pintura, da escultura, da arquitetura etc., sugerindo que imagem se refere a qualquer manifestação numa semiótica plástica. Quando a palavra imagem aparece em estudos de semiótica aplicada a esse domínio da expressão, entende-se imagem como aquilo que se pode ver (PIETROFORTE, 2016).

Segundo Tilly et al. (2010), as demandas dos agentes políticos encontram canais de manifestação e organização nas redes sociais, embora tais avanços tecnológicos tragam desafios. Da mesma forma, simples acessos ou visualizações de conteúdo desencadeiam os mecanismos de seleção, e os usuários estão cada vez mais conscientes de que fazem parte de uma rede que reproduz seu modo de existir (PEDRONI et al., 2014).

A necessidade de comunicação do governo com seus cidadãos, assim como a ocorrência da linguagem não verbal nas plataformas dos portais municipais, tem mostrado que é tarefa da semiótica organizar essas categorias e essas operações abstratas, que estão na base de nossa produção e compressão do sentido (TATIT, 2001).

\section{A pesquisa bibliométrica}

E, para quantificar isso, diversas técnicas analíticas têm sido utilizadas, como mapeamento científico, quantificação de diferentes indicadores bibliométricos, como ano de publicação, idioma, área de publicação, tipo de documentos, organizações, autores, fontes de origem, países e citação, e as estruturas e dinâmicas de desenvolvimento dos termos apresentados (SEGURA-ROBLES et al., 2020).

Foi a partir do início do século XX que a técnica bibliométrica começou a ser utilizada. No início, restringia-se a quantificar obras, livros e volume de palavras. Com a evolução da técnica, passou-se para a análise da produção bibliográfica, como citações e autores em artigos e periódicos e outros documentos (ARAÚJO, 2006). 


\section{METODOLOGIA}

O estudo teve como escopo o levantamento bibliométrico de publicações nacionais e internacionais concernentes ao uso de imagens na prática da comunicação da governança pública nos portais governamentais dos municípios brasileiros e de outros países, com intuito de revelar sobre a abordagem dos temas governança pública, comunicação pública e semiótica em pesquisas científicas. Em virtude do campo de abrangência das bases utilizadas, optou-se pela busca por artigos nacionais e internacionais, cuja amostra contemplem, tanto os portais governamentais dos municípios brasileiros, quanto os de outros países. Em estudos bibliométricos dessa natureza são examinados artigos publicados procurando identificar relações entre autores e temáticas na pesquisa (FERREIRA et al., 2013).

\section{Caracterização da pesquisa}

Este levantamento tem por objetivo também caracterizar a evolução dos estudos bibliométricos nas áreas temáticas vinculadas a public communication, public governance, semiotics e image(s), das bases de dados Eric - Educational Resources Information Centre, Google Scholar, Science Direct, Scopus e Web of Science.

Esta pesquisa é caracterizada como bibliométrica. Quanto aos seus objetivos, é definida como exploratório-descritiva. Este tipo de pesquisa envolve o levantamento bibliográfico e documental, entrevistas não padronizadas e estudos de caso (GIL, 2008).

Em relação à sua natureza, é considerada uma pesquisa aplicada, uma vez que visa a aplicabilidade prática, apontando a solução de problemas específicos, envolvendo verdades e interesses locais (GIL, 2010).

Necessário se faz o conhecimento dos critérios que caracterizam a pesquisa realizada. Encontram-se na literatura, três leis bibliométricas, sendo a Lei de Lotka, que está relacionada a produtividade científica, a mais utilizada. Há também a Lei de Bradford, relacionada à dispersão da produção científica; e a Lei de Zipf, que refere à ocorrência de palavras no texto. A presente pesquisa utiliza-se da Lei de Zipf, já que reflete a medição da frequência do aparecimento das palavras em diversos artigos (VANTI, 2002).

Em virtude do vínculo com a Administração Pública enquanto ciência social aplicada, a área de conhecimento selecionada foi a Business, Management and Accounting, em razão do amplo espectro das temáticas abordadas e seu vínculo com Negócios e Gestão (BULGACOV et al., 2010).

\section{Procedimento de coleta e análise dos dados}

A busca junto às bases de dados Eric, Google Scholar, Science Direct, Scopus e Web of Science se fez em razão de obter respostas mais abrangentes e robustas para a pesquisa realizada. A compilação e análise dos dados para a pesquisa foi realizada em julho de 2020. A busca foi realizada somente por artigo, junto ao título, palavras-chave e resumo, em idioma inglês para maior alcance das bases de dados.

Optou-se pela base de dados Education Resources Information Center (ERIC) por ela melhor atender a critérios de inclusão, descritores que refinam as buscas, já que se destina a oferecer acesso ilimitado a mais 
de 1,4 milhões de registros bibliográficos da área de Educação e de áreas relacionadas. É possível inferir que os mecanismos de busca na base de dados são mais eficientes do que os de cada periódico nacional, visto que a ERIC possui, além da busca por palavras-chave, os descritores que favorecem o refinamento da seleção (BOMFIM et al., 2016).

Quanto a opção pelo Google Scholar, no geral estudos mostram que ele é mais simples e mais conveniente de usar do que, por exemplo, ferramentas de pesquisa federadas ou sistemas de descoberta de bibliotecas. Utiliza-se o Google Scholar principalmente para encontrar documentos de texto completo, artigos de periódicos e recursos em inglês. Em estudos mais recentes, verificou-se que a plataforma obteve resultados mais relevantes (OH et al., 2019).

A razão da opção pela Web of Science (WoS) justifica-se por ser uma das principais bases de dados científicas. Ressalta-se que a WoS é a principal coleção da Thomson Reuters, New York, uma vez que com as demais poderia incorrer em incongruências de resultados em termos de idioma, conteúdo geral e rastreabilidade. Por estas razões, Current Contents Connect, Derwent Innovations Index, Base de dados de periódicos coreanos $(\mathrm{KCl})$, Medline, Russian Science Citation Index e SciELO Citation Index não foram considerados para este levantamento bibliométrico (ADDOLORATO et al., 2019).

A Thomson possui um renomado fator de impacto nos Estados Unidos e Europa. Por outro lado, a opção pelo uso da base Scopus se dá pela grande relevância e um amplo banco de dados de periódicos e de citações disponíveis. Cada uma delas tem um fator de impacto importante e diferente (ANKER et al., 2019).

A Science Direct é um banco de dados de periódicos interdisciplinares editados pela Elsevier, que possui extensa gama de publicações científicas, bases de dados e livros. A influência anglo-saxônica neste caso pode resultar em um retorno maior de publicações no idioma inglês, que é a língua de preferência da comunidade científica (DUARTE et al., 2018).

O pressuposto metodológico remete à ideia de análise visual, visto que notadamente os artigos que esperam ser encontrados dentro dos parâmetros e objetivos da pesquisa possam apresentar imagens em seus textos. Contudo, não se vislumbra a metodologia, natureza ou parâmetros das imagens utilizadas nos resultados, mas sim a ocorrência de palavras e expressões que caracterizam o levantamento bibliométrico de publicações.

Tabela 1: Termos de pesquisa.

\begin{tabular}{|l|l|l|}
\hline Database & Search Field Type & Search \\
\hline Eric & $\begin{array}{l}\text { Title, Abstract or Author, } \\
\text { Keywords }\end{array}$ & "public communication" "public governance" "semiotics" "image" "images" \\
\hline $\begin{array}{l}\text { Google } \\
\text { Scholar }\end{array}$ & Title, Abstract, Keywords & "public communication" "public governance" image AND OR images semiotics \\
\hline $\begin{array}{l}\text { Science } \\
\text { Direct }\end{array}$ & $\begin{array}{l}\text { Title, Abstract or Author- } \\
\text { specified Keywords }\end{array}$ & $\begin{array}{l}\text { "public communication" AND "public governance" AND (image AND images) AND } \\
\text { semiotics }\end{array}$ \\
\hline Scopus & $\begin{array}{l}\text { Article title, Abstract, } \\
\text { Keywords }\end{array}$ & $\begin{array}{l}\text { (TITLE-ABS-KEY (image?) AND TITLE-ABS-KEY ("public governance") AND TITLE-ABS- } \\
\text { KEY ("public communication") AND TITLE-ABS-KEY (semiotics)) }\end{array}$ \\
\hline $\begin{array}{l}\text { Web of } \\
\text { Science }\end{array}$ & Title, Abstract, Keywords & "public communication" AND "public governance" AND image* AND semiotics \\
\hline
\end{tabular}

A metodologia tratou de identificar os artigos publicados nas bases e os journals que publicaram sobre o assunto, cujo escopo possuam os termos 'public communication', 'public governance', 'semiotics', 
'image' e 'images', de acordo com a Tabela 1, e com o uso do operador booleano 'e'. Não foi utilizado nenhum recorte temporal, e o tipo de documento selecionado foi artigo. Neste sentido, excluídos os artigos duplicados, para elegibilidade dos documentos encontrados junto aos objetivos do estudo, a triagem realizada a partir do emprego dos termos de pesquisa se faz pela leitura dos resumos e, uma vez caracterizados, serão confirmados posteriormente pela sua leitura na íntegra.

Foi realizada a pesquisa empregando também um número inferior de combinações de termos e expressões, com o objetivo de também abranger publicações reveladas pelo escopo sem que estivessem limitadas à busca com a totalidade dos termos. A seguir, serão apresentados e discutidos os resultados obtidos.

\section{RESULTADOS E DISCUSSÃO}

Após a tabulação, é realizada a caracterização e análise e do levantamento bibliométrico a partir dos recursos metodológicos empregados pelos pesquisadores. Essa análise que validará como produção científica sobre o uso de imagens na prática da comunicação da governança pública nos portais governamentais dos municípios brasileiros (sites das prefeituras) e de outros países, junto às bases de dados Eric, Google Scholar, Science Direct, Scopus e Web of Science.

A caracterização inicia sintetizando o conjunto de artigos encontrados em relação aos termos de busca utilizados, assim como o ano e o journal de publicação, até a data de 20 de julho de 2020. Na Tabela 2, foi possível identificar a totalidade de 80 artigos publicados a partir da combinação dos termos de pesquisa. Conforme é possível visualizar, a combinação dos termos e expressões "Public Communication; Public Governance; Semiotics; Image(s)" e "Public Governance; Semiotics;" não retornaram qualquer resultado.

Tabela 2: Relação de artigos, anos e journal de publicação.

\begin{tabular}{|c|c|c|c|c|}
\hline Termos & $\mathbf{N}$ & Título & Ano & Journal \\
\hline \multirow{8}{*}{$\begin{array}{l}\text { Public } \\
\text { Communication; } \\
\text { Semiotics; } \\
\text { Image(s) }\end{array}$} & 1 & A imagem epistémica entre o saber e o fazer crer & 2013 & CECS/Universidade do Minho \\
\hline & 2 & $\begin{array}{l}\text { Communicating model uncertainty for natural hazards: A } \\
\text { qualitative systematic thematic review }\end{array}$ & 2019 & $\begin{array}{l}\text { International Journal of Disaster } \\
\text { Risk Reduction }\end{array}$ \\
\hline & 3 & $\begin{array}{l}\text { Correlation of the concepts of public communication and PR- } \\
\text { discourse in the mass media space }\end{array}$ & 2016 & $\begin{array}{l}\text { Indian Journal of Science and } \\
\text { Technology }\end{array}$ \\
\hline & 4 & $\begin{array}{l}\text { Holistic strategy of public action and visual digitalization in } \\
\text { convergent environment }\end{array}$ & 2010 & Observatorio (OBS) \\
\hline & 5 & $\begin{array}{l}\text { Journalism Research in Germany: Origins, theoretical } \\
\text { innovations and future outlook }\end{array}$ & 2006 & Brazilian Journalism Research \\
\hline & 6 & $\begin{array}{l}\text { Para doar sangue, você precisa conhecer a pessoa? Estratégias } \\
\text { de polidez em campanhas brasileiras de doação de sangue: uma } \\
\text { análise multimodal }\end{array}$ & 2016 & Linguagem em (Dis)curso \\
\hline & 7 & $\begin{array}{l}\text { The communication of forensic science in the criminal justice } \\
\text { system: A review of theory and proposed directions for } \\
\text { research }\end{array}$ & 2015 & Science \& Justice \\
\hline & 8 & $\begin{array}{l}\text { Towards a transformative image of Arab women in anti- } \\
\text { violence adverts: Semiotics analysis of Arab social media } \\
\text { adverts }\end{array}$ & 2019 & Media Watch \\
\hline \multirow{3}{*}{$\begin{array}{l}\text { Public } \\
\text { Governance; } \\
\text { Semiotics; } \\
\text { Image(s) }\end{array}$} & 9 & $\begin{array}{l}\text { A metafunção composicional em charges de futebol: um estudo } \\
\text { do espaço visual à luz da Gramática do Design Visual }\end{array}$ & 2016 & $\begin{array}{l}\text { Revista Pesquisas em Discurso } \\
\text { Pedagógico }\end{array}$ \\
\hline & 10 & $\begin{array}{l}\text { Complex territories, complex circulations: The pacification of } \\
\text { the Complexo do Alemão in Rio de Janeiro }\end{array}$ & 2015 & $\begin{array}{l}\text { John Benjamins Publishing } \\
\text { Company }\end{array}$ \\
\hline & 11 & $\begin{array}{l}\text { Please don't feed the models: Blogues da Ana e os limites da } \\
\text { comunicação pública }\end{array}$ & 2019 & $\begin{array}{l}\text { Eikon Journal on Semiotics and } \\
\text { Culture }\end{array}$ \\
\hline Public & 12 & Democratic Crisis or Return to the Western World? Civic & 2018 & Lituanus \\
\hline
\end{tabular}


Communication;

Public

Governance;

Image(s)

Engagement, Social Capital, and Media in Latvia.

13 Governance, participation and avoidance: everyday public

involvement in the Scottish NHS

14 Implied truth, complementary media practices, and successful

atomized activism in China

15 Public relations, discourse practice and the public interest:

analysis of a health communication campaign

16 The politics and poetics of migrant narratives

17 Human Rights and the Critiques of the Public-private Distinction

Public

Communication;

Public

Governance;

Semiotics;

Public

Communication:

Semiotics;

Public

Governance,

Image(s)
18 Quasi-Governmental Organizations at the Local Level: Publicly-

Appointed Directors Leading Nonprofit Organizations

The accounting-globalisation interrelation: An overview with

19 some reflections on the neglected dimension of emancipatory potentiality

20 Declaring Talloires: Profile of sustainability communications in

20 Australian signatory universities

21 Election poster busting. Forms and functions of a (new) blend of text pattern

22 Legeuphemism as a special case of euphemism

23 Modo simbolico, mondi possibili e matematica

24 On the correlation of genres of natural and artistic discourses:

24 Setting the problem (in the genre "Letter to the editor")

25 Reestablishing a public space and promoting the debate without taking a position

Semiotics of the "third force": Most and the performative and

26 visual dimension of political life in the post-electoral period in croatia, 2015

The Nation Brand Regime: Nation Branding and the Semiotic

27 Regimentation of Public Communication in Contemporary Macedonia

28 Viral communication and the formation of counter-publics

29 Community branding and brand images in Ontario, Canada

30 Destination Marketing Organizations and destination

30 marketing: A narrative analysis of the literature

31 Factors shaping urban greenspace provision: A systematic review of the literature

32 More Talk than Walk? UMNO, New Politics and Legitimation in Contemporary Malaysia

33 Political-Legal Manipulation as the Basis for the Governance of

33 Society in the era of pos-truth

34 Public Governance and Strategic Intelligence Innovation in the

34 Public Service

35 Public Governance in the MENA Region: Reform Trends and Patterns

36 Reforms and identities How relentless pursuit of improvements

produces a sense of helplessness among bureaucrats

37 Understanding shortages of sufficient health care in rural areas

38 What is the private sector? Understanding private provision in

38 the health systems of low-income and middle-income countries

39 A novel chaotic key-based algorithm for still images

A novel parallel image encryption with chaotic windows based on logistic map

41 A Simple Intervention to Reduce Framing Effects in Perceptions

41 of Global Climate Change

Public

Communication; Image(s)
42 Affect, risk perception, and the use of cigarettes and e-

42 cigarettes: A population study of U.S. adults

43 Analyzing the factors that influence U.S. public support for exporting natural gas

44 Authenticity and tolerance: Virtues reframed for defining

44 common ground in communication

45 Brand management in terrorism: the case of Hezbollah
Edinburgh Research Archiv

2012 Edinburgh Research Archiv

2020 Global Media and China

2013 RMIT University

2020 European Journal of Cultural Studies

2010 Vrije Universiteit Amsterdam

2016 De Gruyter

2006 Critical Perspectives on

Accounting

2018 Journal of Cleaner Production

2018 Zeitschrift fur Angewandte

2015 Sibirskii Filologicheskii Zhurnal

Bollettino della Unione

2006

Matematica Italiana

Vestnik Tomskogo

2016 Gosudarstvennogo Universiteta

2013 Bulletin Suisse de Linguistique Appliquee

2019 Narodna Umjetnost

2016 Signs and Society

2016 Lexia

2015 Place Branding and Public

Diplomacy

2014 Tourism Management

2018 Landscape and Urban Planning

2013 Journal of Contemparary Asia

2019

Vestnik Tomskogo

Gosudarstvennogo Universiteta

Revista História-Debates e

Tendências

International Journal of Public Administration

2017 Journal of Organizational Change Management

2014 Health Policy

2016 The Lancet

International Journal of

2017 Computational Science and Engineering

2017 Computers and Electrical Engineering

2017 Environmental Communication

2018 BMC Public Health

2018 Energy Policy

2010 International Journal of the Humanities

2018 Journal of Policing, Intelligence 


\begin{tabular}{|c|c|c|c|}
\hline & & & and Counter Terrorism \\
\hline 46 & $\begin{array}{l}\text { Climate change discourse and the shaping of meanings in } \\
\text { Lithuanian mass media }\end{array}$ & 2009 & Filosofija, Sociologija \\
\hline 47 & Ethical aspects of gender stereotypes in romanian advertising & 2016 & $\begin{array}{ll}\text { Vestnik } & \text { Tomskogo } \\
\text { Gosudarstvennogo Universiteta }\end{array}$ \\
\hline 48 & $\begin{array}{l}\text { Ethics in fMRI studies: A review of the EMBASE and MEDLINE } \\
\text { literature }\end{array}$ & 2008 & Clinical Neuroradiology \\
\hline 49 & Examining "the heart of the west" & 2009 & Public Historian \\
\hline 50 & $\begin{array}{l}\text { Exploring a black box: Cross-national study of visit effects on } \\
\text { visitors to large physics research centers in Europe }\end{array}$ & 2009 & Science Communication \\
\hline 51 & $\begin{array}{l}\text { Extreme rhetoric in the } 2004 \text { presidential campaign: Images of } \\
\text { geopolitical and regional division }\end{array}$ & 2005 & Canadian Slavonic Papers \\
\hline 52 & $\begin{array}{l}\text { Hide text depending on the three channels of pixels in color } \\
\text { images using the modified LSB algorithm }\end{array}$ & 2020 & $\begin{array}{l}\text { International Journal of Electrical } \\
\text { and Computer Engineering }\end{array}$ \\
\hline 53 & $\begin{array}{l}\text { Image and substance: From symbolic to behavioral } \\
\text { relationships }\end{array}$ & 1993 & Public Relations Review \\
\hline 54 & $\begin{array}{l}\text { Image events, the public sphere, and argumentative practice: } \\
\text { The case of radical environmental groups }\end{array}$ & 2003 & Argumentation \\
\hline 55 & $\begin{array}{l}\text { Images of state school system administration in the literature } \\
\text { on educational administration: An australian perspective }\end{array}$ & 1982 & \begin{tabular}{|l|l|} 
Journal of \\
Administration
\end{tabular} \\
\hline 56 & $\begin{array}{l}\text { InfoRAD: computers for clinical practice and education in } \\
\text { radiology. Teleradiology, information transfer, and PACS: } \\
\text { implications for diagnostic imaging in the 1990s. }\end{array}$ & 1993 & $\begin{array}{l}\text { Radiographics - Radiological } \\
\text { Society of North America }\end{array}$ \\
\hline 57 & $\begin{array}{l}\text { Interpretation of forest characteristics from computer- } \\
\text { generated images }\end{array}$ & 2007 & Landscape and Urban Planning \\
\hline 58 & $\begin{array}{l}\text { Journalists' views about reporting avian influenza and a } \\
\text { potential pandemic: A qualitative study }\end{array}$ & 2012 & $\begin{array}{l}\text { Influenza and other Respiratory } \\
\text { Viruses }\end{array}$ \\
\hline 59 & $\begin{array}{l}\text { Lifeguarding Operational Camera Kiosk System (LOCKS) for flash } \\
\text { rip warning: Development and application }\end{array}$ & 2019 & Coastal Engineering \\
\hline 60 & Moralizing in public communication & 2015 & \begin{tabular}{|lr} 
Lili $\quad$ Zeitschrift & fur \\
Literaturwissenschaft & und \\
Linguistik & \\
\end{tabular} \\
\hline 61 & $\begin{array}{l}\text { Narratives and images used by public communication } \\
\text { campaigns addressing social determinants of health and health } \\
\text { disparities }\end{array}$ & 2012 & $\begin{array}{l}\text { Journal of Environmental } \\
\text { Research and Public Health }\end{array}$ \\
\hline 62 & $\begin{array}{l}\text { Novel Variants of a Histogram Shift-Based Reversible } \\
\text { Watermarking Technique for Medical Images to Improve Hiding } \\
\text { Capacity }\end{array}$ & 2017 & Journal of Healthcare Engineering \\
\hline 63 & PETA making social noise: A perspective on shock advertising & 2013 & \begin{tabular}{|l}
$\begin{array}{l}\text { Portuguese Journal of Social } \\
\text { Science }\end{array}$ \\
\end{tabular} \\
\hline 64 & Public perception of space in Japan: Media and space & 2002 & Hermes \\
\hline 65 & $\begin{array}{l}\text { Resistance and Renewal: Ottoman Armenian 'Soldiers' } \\
\text { Photography' during the First World War }\end{array}$ & 2018 & Zeitgeschichte \\
\hline 66 & $\begin{array}{l}\text { Sensitizing the concept of mediatization for the study of social } \\
\text { movements }\end{array}$ & 2019 & Communications \\
\hline 67 & $\begin{array}{l}\text { Shaping the urban life of oil in Bahrain: Consumerism, leisure, } \\
\text { and public communication in manama and in the oil camps, } \\
\text { 1932-1960s }\end{array}$ & 2013 & $\begin{array}{l}\text { Comparative Studies of South } \\
\text { Asia, Africa and the Middle East }\end{array}$ \\
\hline 68 & $\begin{array}{l}\text { Social image of nursing and public communication satrategies } \\
\text { to obtain a positive image }\end{array}$ & 2011 & Index de Enfermeria \\
\hline 69 & $\begin{array}{l}\text { South Koreans' perceptions of North Koreans and implications } \\
\text { for public relations campaigns }\end{array}$ & 2004 & Public Relations Review \\
\hline 70 & $\begin{array}{l}\text { Synchronization of uncertain constrained hyperchaotic systems } \\
\text { and chaos-based secure communications via a novel } \\
\text { decomposed nonlinear stochastic estimator }\end{array}$ & 2016 & Nonlinear Dynamics \\
\hline 71 & $\begin{array}{l}\text { Television Coverage of Jesse Jackson's Speech to the } 1984 \\
\text { Democratic National Convention }\end{array}$ & 1988 & \begin{tabular}{|l|l|l|l} 
Journal of Broadcasting \& \\
Electronic Media
\end{tabular} \\
\hline 72 & $\begin{array}{l}\text { Television news and public knowledge: Understanding the } \\
\text { economy }\end{array}$ & 1997 & Hermes \\
\hline 73 & Terrae animatae: metamorphic maps on postcards & 1994 & Map Collector \\
\hline 74 & $\begin{array}{l}\text { Text embedded into encrypted image based on genetic } \\
\text { algorithm on piece-wise linear chaotic map }\end{array}$ & 2016 & $\begin{array}{l}\text { Indian Journal of Science and } \\
\text { Technology }\end{array}$ \\
\hline 75 & $\begin{array}{l}\text { The energy question in the Belgian daily press during 2010: The } \\
\text { role of region, newspaper type and newspaper section }\end{array}$ & 2015 & $\begin{array}{l}\text { Journal of Science } \\
\text { Communication }\end{array}$ \\
\hline 76 & The Map, the Other and the public visual image & 2015 & Social and Cultural Geography \\
\hline 77 & Visual culture and politicisation in 19th-century Europe & 2017 & Passato e Presente \\
\hline
\end{tabular}




\begin{tabular}{|l|l|l|l|l|} 
& 78 & $\begin{array}{l}\text { Visual framing of European Years in Romanian public } \\
\text { communication campaign blogs }\end{array}$ & 2014 & Communication and Society \\
\cline { 2 - 5 } & 79 & $\begin{array}{l}\text { Visual Persuasion in George W. Bush's Presidency: Cowboy } \\
\text { Imagery in Public Discourse }\end{array}$ & 2011 & Congress and the Presidency \\
\cline { 2 - 5 } & 80 & $\begin{array}{l}\text { Where the location trumpets, freedom goes down the drain: } \\
\text { Images of interurban competition using the representation of } \\
\text { Leipzig as a "Candidate City" for the Olympic Games 2012 }\end{array}$ & 2005 & Social Geography \\
\hline $\begin{array}{l}\text { Public } \\
\begin{array}{l}\text { Communication; } \\
\text { Public } \\
\text { Governance; } \\
\text { Semiotics; } \\
\text { Image(s) }\end{array}\end{array}$ & & & & \\
\hline $\begin{array}{l}\text { Public } \\
\text { Governance; } \\
\text { Semiotics; }\end{array}$ & & & & \\
\hline
\end{tabular}

Quanto à análise das publicações, na descrição metodológica deste estudo optou-se pela Lei bibliométrica de Zipf, que refere à ocorrência de palavras no texto. A tabulação dos resultados obtidos separou os artigos por título, ano de publicação e revistas científicas. Como se pode depreender da Tabela 2, não se vislumbrou publicações atinentes ao escopo com a totalidade dos termos empregados.

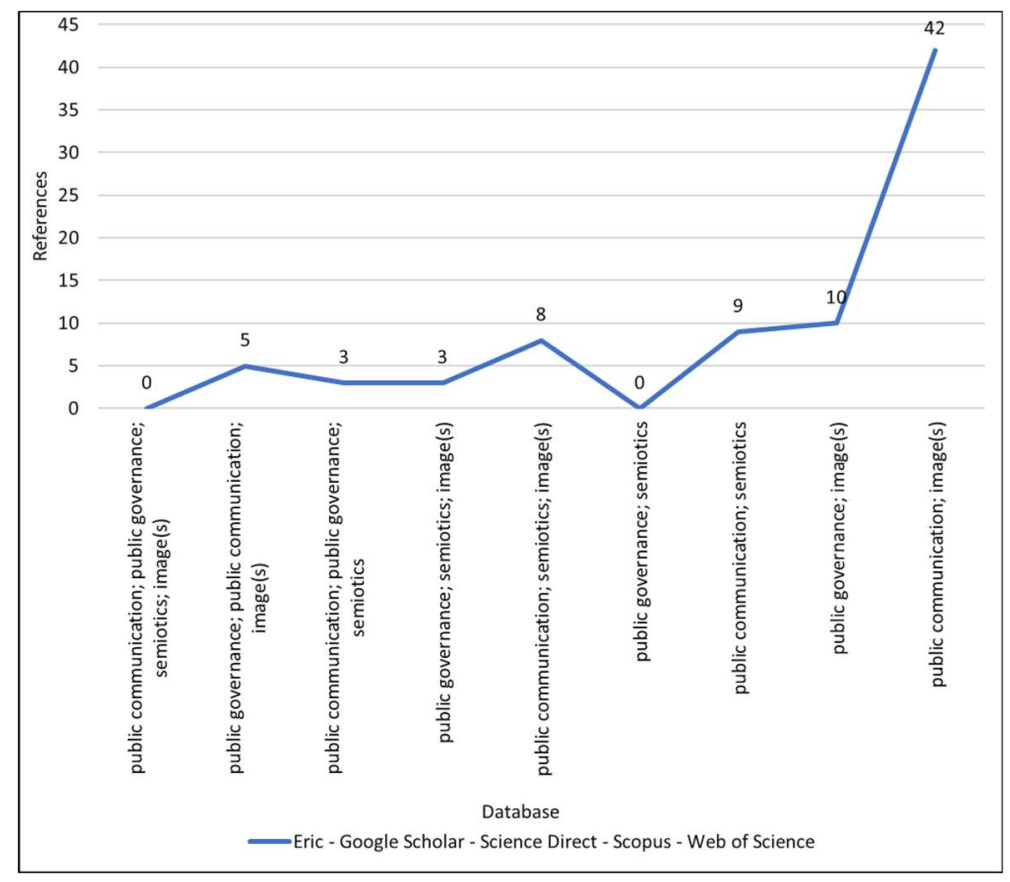

Figura 1: Quantidade de publicações por termos de busca.

Assim sendo, como já tabelado, a Figura 1 demonstra que com o emprego da totalidade dos termos de pesquisa, quais são Public Communication, Public Governance, Semiotics e Image(s), encontrou-se 0 resultado nas cinco bases de dados utilizadas. 0 mesmo resultado 0 foi encontrado com o uso dos termos Public Governance e Semiotics. Já Public Governance, Public Communication e Image(s) retornaram 5 referências. As buscas utilizando os termos Public Communication, Public Governance e Semiotics, assim como com os termos Public Governance, Semiotics e Image(s), obtiveram 3 referências cada. Na sequência, Public Communication, Semiotics e Image(s) (8); Public Communication e Semiotics (9); Public Governance, Public Communication e Image(s) (10). Por fim, Public Communication, e Image(s) retornaram 42 referências. Segundo a Lei de Zipf, que refere à ocorrência de palavras e expressões no texto, retornou um 
número significativo de resultados quando do uso da combinação de poucos termos, sobretudo com o uso de Public Communication, e Image(s) que, juntas, retornaram 42 artigos. Essa fragmentação se fez importante a fim de mapear possíveis publicações dentro do escopo, mas que não foram alcançadas por todos os termos e expressões propostos pela pesquisa.

Um dos aspectos de pesquisa que se vislumbrou diz respeito à quantidade de produção por ano. $\mathrm{A}$ se considerar a totalidade de publicações encontradas (80), notadamente a partir de 2015 (08) encontra-se uma tendência crescente de publicações. De 1982 a 2012, houve uma oscilação entre 1 e 3 publicações, o que contrasta com a quantidade de resultados encontrados a partir de então. Isso mostra maior evidência dos termos pesquisados na produção científica. A Figura 2 demonstra as publicações mapeadas. Cabe ressaltar que a compilação dos dados para a pesquisa foi realizada em julho de 2020.

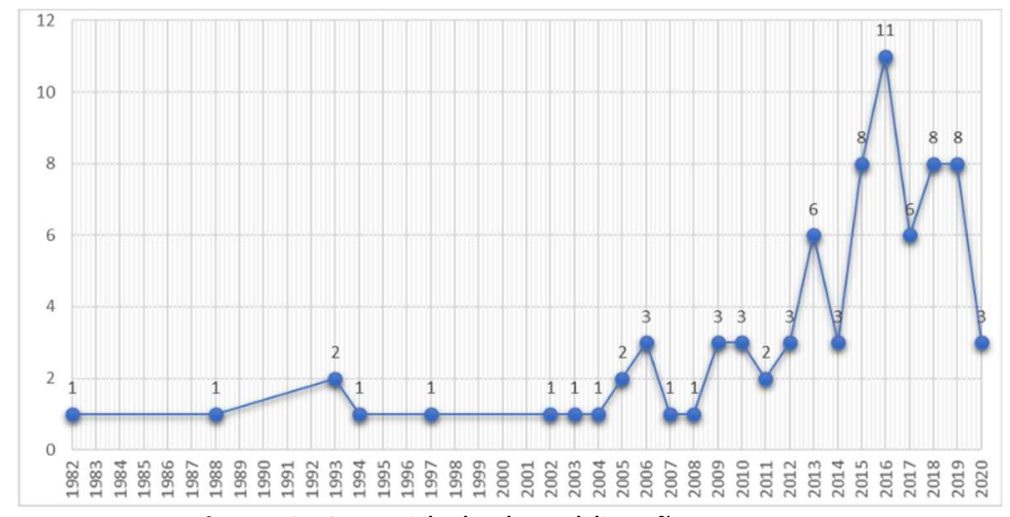

Figura 2: Quantidade de publicações por ano.

A partir dos anos 2013 (6) e 2015 (08) são verificados sobressaltos e uma tendência ascendente de publicações anuais sobre os temas pesquisados. Não se optou pelo critério de quantidade de autores por artigo ou a classificação de revistas por publicação nesta abordagem do problema.

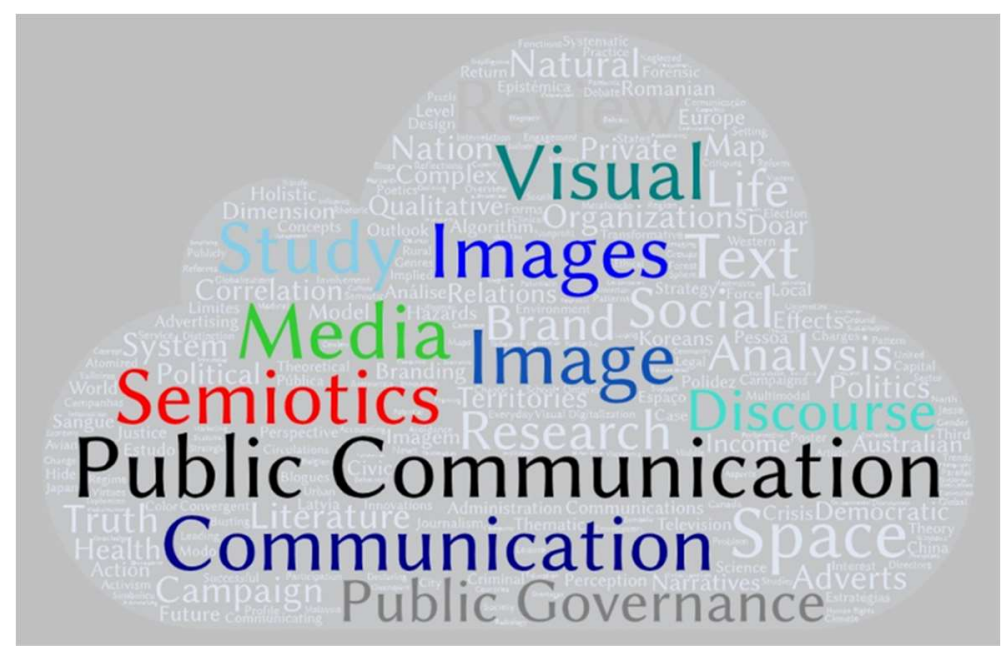

Figura 3: Nuvem de palavras dos artigos mapeados.

Ao realizar uma lista hierarquizada de ocorrência de palavras, utilizou-se termos pertencentes não somente ao escopo da pesquisa, como também aos títulos e às palavras-chave dos artigos encontrados, de modo a mapear eventuais publicações atinentes ao tema, mas não encontradas a partir dos termos de pesquisa. Há destaque na repetição das palavras Public Communication, Public Governance, Semiotics e 
Images. Em relação ao aparecimento de palavras alheias aos termos de escopo, destacam-se Visual, Media e Discourse. A Figura 3 demonstra estes achados por meio de uma nuvem de palavras.

A partir da nuvem de palavras, dado o amplo espectro de palavras alcançadas, observa-se que a interdisciplinaridade presente neste levantamento serve como um elo entre as temáticas abordada e as suas similitudes verificadas a partir das técnicas bibliométricas junto às diferentes ciências neste levantamento.

\section{CONCLUSÕES}

O presente levantamento objetivou analisar a literatura a partir da temática do uso de imagens na prática da comunicação da governança pública nos portais governamentais dos municípios brasileiros e de outros países. E, dessa forma, proceder à recolha de informações de relação mútua dos temas governança pública, comunicação pública e semiótica em pesquisas científicas.

Os resultados encontrados a partir das coletas de dados e das análises conduzidas, ainda que considerando o grande espectro da área de conhecimento que é a Administração Pública (Business, Management and Accounting), não apontam qualquer produção científica, atributo ou mesmo propriedade que remete à investigação inerente ao escopo da pesquisa. Embora as buscas não se restringissem à totalidade dos termos, os resultados sugerem que o emprego de um número inferior de combinações também não retorne resultados pertinentes ao contexto de pesquisa.

Os resultados também sugerem que merece destaque o volume significativo de publicações que retornaram a partir da busca com os termos comunicação pública e imagens, assim como a tendência de crescimento da produção científica com o emprego de todos os termos de pesquisa, verificado a partir de 2015, apesar, em ambos os casos, de se referirem a produções científicas alheias ao tema de pesquisa. Dada a inexistência de resultados de artigos com o emprego simultâneo de todos os termos de pesquisa, de modo semelhante, os termos Governança Pública e Semiótica não obtiveram retorno algum.

A qualidade distintiva que a governança pública e a comunicação pública possuem mostram se fundamentais como temática para a formulação e implementação de políticas públicas. Nesse sentido, esse déficit científico apontado no presente trabalho pode ser revelador de uma menor percepção dos cidadãos em relação ao conteúdo dos portais governamentais dos municípios (sites de prefeituras), bem como sobre a comunicação da capacidade da ação do Estado.

Como limitação do estudo é possível destacar sobre a crença de que as únicas formas de interpretação e conhecimento do mundo são aquelas manifestadas pela linguagem verbal oral ou escrita, em detrimento da linguagem não-verbal, como pinturas, desenhos, ou a representação por imagens de modo geral. Importante se faz esse destaque, já que é dessa forma intricada de comunicação e de significação que se encarrega a semiótica, que é a ciência tem por objeto a investigação destas linguagens. Considerando a amplitude de significação e o quão vasto é o campo de estudo da semiótica, os resultados também sugerem que não seria pelo aumento do número de bases de dados ou robustez da amostra, que a produção científica seja mais evidente em levantamentos bibliográficos dessa natureza. 


\section{REFERÊNCIAS}

ADDOLORATO, S.; CALABUIG, F.; PRADO-GASCÓ, V.; GALLARDO, L.; GARCÍA-UNANUE, J.. Bibliometric analysis of fitness equipment: how scientific focuses affect life-cycle approaches and sustainable ways of development. Sustainability, v.11, n.5728, 2019. DOI: https://doi.org/10.3390/su11205728

ANKER, M. S.; HADZIBEGOVIC, S.; LENA, A.; HAVERKAMP, W.. The difference in referencing in Web of Science, Scopus, and Google Scholar. ESC Heart Failure, v.6, n.6, p.1291-1312, 2019.

ARAÚJO, C. A.. Bibliometria: evolução histórica e questões atuais. Em Questão, v.12, n.1, 2006.

BULGACOV, S.; CANHADA, D. I. D.; BULGACOV, Y. L. M.. A comparação dos processos de acompanhamento acadêmico e de ensino de Administração em duas universidades brasileiras e duas estrangeiras. Revista Administração: Ensino e Pesquisa, v.11, n.3, p.355-372, 2010 DOI: https://doi.org/10.13058/raep.2010.v11n3.134

BOMFIM, A. B. C.; SILVA, S. A. P. S.; MIRANDA, M. L. J.. A produção do conhecimento sobre a formação continuada de professores de Educação Física: uma análise entre estudos nacionais e internacionais. Journal of Physical Education, v.27, n.1, 2016.

DALLARI, D. A.. Elementos de teoria geral do estado. 24 ed. São Paulo: Saraiva, 2003.

DECKERT, C. T.. A Comunicação Pública por Meio da Internet em Programas de Desenvolvimento Brasileiros em promoção da cidadania. Revista Mediação, Belo Horizonte, v.19, n.24, 2017.

DUARTE, H.; ALFONSO, W.. Revisión bibliométrica sobre la enseñanza-aprendizaje de Ergonomía en las modalidades virtual y distancia. Educación Médica Superior, v.32, n.4, p.184-198, 2018.

DUARTE, J.. Comunicação Pública: estado, mercado, sociedade e interesse público. 3 ed. São Paulo: Atlas, 2012.

GIL, A. C.. Como elaborar projetos de pesquisa. 5 ed. São Paulo: Atlas. 2010.

GIL, A. C.. Métodos e técnicas de pesquisa social. 6 ed. São Paulo: Atlas, 2008.

FERREIRA, M. A. S. P. V.; PINTO, C. S. F.; SERRA, F. A. R.; SANTOS, J. C.. Um estudo bibliométrico da contribuição de John Dunning na pesquisa em negócios internacionais. Revista Brasileira de Gestão de Negócios, v.15, n.46, p.5675, 2013. DOI: https://doi.org/10.7819/rbgn.v15i46.1163
HASWANI, M. F.. Comunicação pública: bases e abrangências. São Paulo: Saraiva, 2013.

MATIAS-PEREIRA, J.. Governança no setor público. São Paulo: Atlas, 2010.

MATIAS-PEREIRA, J.. Curso de administração pública: foco nas instituições e ações governamentais. 4 ed. São Paulo: Atlas, 2014.

OH, K. E.; COLÓN-AGUIRRE, M.. A comparative study of perceptions and use of Google Scholar and Academic Library Discovery Systems. College \& Research Libraries, v.80, n.6, p.876, 2019. DOI: https://doi.org/10.5860/crl.80.6.876

PIETROFORTE, A. V.. Análise do texto visual: a construção da imagem. 2 ed. São Paulo: Contexto, 2016.

PEDRONI, M.; PASQUALI, F.; CARLO, S.. My friends are my audience: mass-mediation of personal content and relations in Facebook. Observatório, v.8, n.3, p.97-113, 2014. DOI: http://dx.doi.org/10.15847/obsOBS832014739

SANTAELLA, L.. A cultura das mídias. São Paulo: Razão Social, 1992.

SANTAELLA, L.. Comunicação e semiótica. São Paulo: Hacker Editores, 2004.

SOUZA, S. M. R.; SANTARELLI, C. P. G.. Contribuições para uma história da análise da imagem no anúncio publicitário. Intercom - Revista Brasileira de Ciências da Comunicação, São Paulo, v.31, n.1, p.133-156, 2008.

SEGURA-ROBLES, A.; MORENO-GUERRERO, A.-J.; PARRAGONZÁLEZ, M.-E.; LÓPEZ-BELMONTE, J.. Review of Research Trends in Learning and the Internet in Higher Education. Social Sciences, v.9, n.6, p.101, 2020. DOI: https://doi.org/10.3390/socsci9060101

TATIT, L.. Análise semiótica através das letras. São Paulo: Ateliê, 2001.

TILLY, C.; WOOD, L. J.. Los movimientos sociales, 1768-2008: desde sus orígenes a Facebook. Barcelona: Crítica, 2010.

WATANUKI, H. M.; NADAE, J.; CARVALHO, M. M.; MORAES, R. O.. Gestão de projetos internacionais: um estudo bibliométrico. Gestão \& Produção, v.21, n.3, p.660-675, 2014. DOI: https://doi.org/10.1590/0104-530X394

VANTI, N. A. P.. Da bibliometria à webometria: uma exploração conceitual dos mecanismos utilizados para medir o registro da informação e a difusão do conhecimento. Ciência da Informação, v.31, n.2, p.152-162, 2002. DOI: https://doi.org/10.1590/S0100-19652002000200016

A CBPC - Companhia Brasileira de Produção Científica (CNPJ: 11.221.422/0001-03) detém os direitos materiais desta publicação. Os direitos referem-se à publicação do trabalho em qualquer parte do mundo, incluindo os direitos às renovações, expansões e disseminações da contribuição, bem como outros direitos subsidiários. Todos os trabalhos publicados eletronicamente poderão posteriormente ser publicados em coletâneas impressas sob coordenação da Sustenere Publishing, da Companhia Brasileira de Produção Científica e seus parceiros autorizados. Os (as) autores (as) preservam os direitos autorais, mas não têm permissão para a publicação da contribuição em outro meio, impresso ou digital, em português ou em tradução. 